\title{
Effects of Viscum coloratum (Kom.) Nakai f. Lutescens Kitag polysaccharide on fertility, longevity and antioxidant capacity of Drosophila melanogaster
}

\author{
Wenbin $\mathrm{YE}^{1 \star}\left(\mathbb{D}\right.$, Long ZHANG ${ }^{1}$, Duli WANG ${ }^{1}$, Yufeng $\mathrm{HE}^{1}$, $\mathrm{Na} \mathrm{LI}^{1}$, Jing JIANG ${ }^{1}$, Yingli $\mathrm{MA}^{1}$
}

\begin{abstract}
The purpose of this study was to investigate the effects of Viscum coloratum (Kom.) Nakai f. Lutescens Kitag polysaccharide on fertility, longevity and antioxidant capacity of Drosophila melanogaster. The Drosophila melanogaster were randomly divided into five groups. Four generations of Drosophila melanogaster were cultured continuously in basic medium with or without polysaccharides $\left(400,200,100\right.$ and $50 \mathrm{mg} / \mathrm{L}$ ). The changes in fertility, lifespan and male-to-female ratio from $F_{1}$ to $F_{4}$ generation. The survival time under acute oxidative damage caused by paraquat and hydrogen peroxide $\left(\mathrm{H}_{2} \mathrm{O}_{2}\right)$ and changes in superoxide dismutase (SOD), catalase (CAT) and malondialdehyde (MDA) content were determined as well. High dose of $V$. coloratum polysaccharide could significantly improve the fertility and lifespans of Drosophila melanogaster from $\mathrm{F}_{1}$ to $\mathrm{F}_{4}$ generation, prolong the survival time under acute oxidative damage. Moreover, the MDA content was decreased sharply, while the activities of SOD and CAT were increased in high group. However, the male-to-female ratio was overall close to 1:1 for F1 to F4 generations among the four groups. V. coloratum polysaccharide improved the antioxidant capacity and vitality of Drosophila melanogaster and promoted their reproductive capacity in a dose-dependent manner.
\end{abstract}

Keywords: Viscum coloratum (Kom.) Nakai f. Lutescens Kitag polysaccharide; Drosophila melanogaster; fertility; longevity; antioxidant capacity.

Practical Application: V. coloratum polysaccharide improved the antioxidant capacity and vitality of D. melanogaster and promoted their reproductive capacity in a dose-dependent manner

\section{Introduction}

Viscum coloratum (Kom.) Nakai, a spermatophyte, is mainly parasitic on mulberry, poplar and other big trees, which is widely distributed in China. It serves as a precious traditional Chinese medicine (TCM) in China's medical prescriptions, which is mainly used for expelling rheumatism, improving one's health and reinforcing liver and kidney. In recent years, it has also been reported that $V$. coloratum (Kom.) Nakai has protection on cough, frostbite, stomach trouble, lumbocrural pain and threatened abortion (Yoo et al., 2019). In the clinic of TCM, $V$. coloratum (Kom.) Nakai f. lutescens Kitag is equivalent to $V$. coloratum (Kom.) Nakai ${ }^{[3]}$. Researches on V. coloratum (Kom.) Nakai medicines and related health products started late in China, which has recently attracted people's attention(Chen et al., 2020).

The main bioactive component in the fruit of $V$. coloratum (Kom.) Nakai f. lutescens Kitag is polysaccharide (V. coloratum polysaccharide), which is mainly composed of highly esterified galactose polymers (Szurpnicka et al., 2019), neutral polysaccharides and acidic polysaccharides (Stein et al., 1999). In recent years, through the optimization of extraction process(Jun \& Yi, 2007) and the study of rheological properties, the research on $V$. coloratum polysaccharide has gradually turned to functional food and pharmacological research. V. coloratum polysaccharide has been used in the treatment of immunocompromised mice and mice with acute alcoholic liver injury, and the immune function and liver injury were recovered well (Ye et al., 2017).

Drosophila melanogaster (D. melanogaster) has the characteristics of simple breeding, rapid reproduction and clear genetic background (Rubin, 1988), which is mostly used in the research of drug and food detection(Jana et al., 2016; Brandt \& Vilcinskas, 2013; Lamas et al., 2016). However, the effects of $V$. coloratum polysaccharide on fertility, longevity and antioxidant capacity of Drosophila melanogaster have not been reported. Therefore, in this study, with D. melanogaster as subject, the effects of $V$. coloratum polysaccharide on fertility, longevity and antioxidant capacity were investigated to provide a theoretical basis for the development of health products and medicines of V. coloratum polysaccharide.

\section{Materials and Methods}

\subsection{Experimental materials and main instruments}

The fruit of V. coloratum (Kom.) Nakai f. lutescens kitag were collected in Liangdang County, (Longnan, China) in July 2018. The commercial kits of Superoxide dismutase (SOD) (lot 
number: 20160822), catalase (CAT) (lot number: 20170509) and MDA (lot number: 20170729) were purchased from Nanjing Jiancheng Bioengineering Institute (Nanjing, China). Yeast extract was purchased from Kmaels Biotechnology Co., Ltd (Shanghai, China). Other instruments used in this study were as follows: thermostatic biochemical incubator (Ningbo Dongnan Instrument Co., Ltd., Ningbo, China), high temperature sterilizer (Shanghai Sanshen Medical Appliance Co., Ltd., Shanghai, China), ultra clean bench (Shandong Biobase Scientific Instrument Co., Ltd., Jinan, China), L5 UV-visible spectrophotometer (INESA Analytical Instrument Co., Ltd., Basil, Switzerland), and high-speed refrigerated centrifuge (Beijing Era Beili Centrifuge Co., Ltd., Beijing, China).

\subsection{Extraction of $V$. coloratum polysaccharides}

The method of extracting polysaccharides from the fruit of $V$. coloratum (Kom.) Nakai f. lutescens kitag mainly referred to the method described in previous studies (Huang \& Huang, 2020). The fruit were dried at $50{ }^{\circ} \mathrm{C}$ and pulverized. After refluxing and degreasing in anhydrous ethanol, it was dried at $50{ }^{\circ} \mathrm{C}$. Then, $100 \mathrm{~g}$ of the dried material was added to $1000 \mathrm{ml}$ of distilled water and heated at $40{ }^{\circ} \mathrm{C}$ for $2 \mathrm{~h}$ to dissolve it. The supernatant obtained by vacuum filtration was concentrated under reduced pressure. The extract was mixed with Sevag reagent in a volume ratio of $1: 5$, and shaken and centrifugation to remove the denatured protein, and this process was repeated 3 times. The obtained crude polysaccharide was dissolved in distilled water at $40{ }^{\circ} \mathrm{C}$, followed by stirring and slowly adding Fehling's reagent simultaneously. After precipitation of the copper complex, the mixture was stood for $1 \mathrm{~h}$, washed with distilled water for 3 times, and centrifuged to obtain the complex. Distilled water at $4{ }^{\circ} \mathrm{C}$ was added into the complex, and then $0.5 \mathrm{~mol} / \mathrm{L}$ $\mathrm{HCl}$ was added dropwise to dissolve all the compound. Next, the crude polysaccharide was precipitated with $95 \%$ ethanol, followed by centrifugation. The obtained floccule was washed with ethanol, acetone and diethyl ether, and freeze-dried into a white polysaccharide powder. Finally, the purity was detected by High performance liquid chromatography - gel permeation chromatography (HPLC-GPC).

\subsection{Determination of fertility}

Wild-type D. melanogaster, provided by the Genetics Laboratory of Northwest Normal University, cultured in a constant temperature and humidity incubator at $25^{\circ} \mathrm{C}$ and $65 \%$ relative humidity. The basic medium for culturing D. melanogaster, including $250 \mathrm{ml}$ of distilled water, $28 \mathrm{~g}$ of corn flour, $22 \mathrm{~g}$ of sucrose, $2.5 \mathrm{~g}$ of agar, $2 \mathrm{ml}$ of propionic acid and $2.5 \mathrm{~g}$ of fresh yeast, was prepared by heating. As previously described (Peng et al., 2009), V. coloratum polysaccharide at concentrations of 50, 100, 200 and $400 \mathrm{mg} / \mathrm{L}$ were added into the basic medium as low, medium and high dose of $V$. coloratum polysaccharide groups, and the basic medium was set as the control group. Specifically, in constant-temperature incubator, 10 pairs of unmated male and female $D$. melanogaster were placed into the medium, and cultured for $7 \mathrm{~d}$. The parents were removed. After the offspring $\left(\mathrm{F}_{1}\right)$ appeared, the number of male and female was counted. After analyzing the recorded data, 10 pairs of unmated $\mathrm{F}_{1}$ male and female were taken into a new medium for subculture, respectively. After cultured for $7 \mathrm{~d}$, the grandchildren $\left(\mathrm{F}_{2}\right)$ were propagated. The $\mathrm{F}_{2}$ generation was further subcultured to reproduce the $\mathrm{F}_{3}$ generation, and the $\mathrm{F}_{3}$ generation was further subcultured to reproduce the $\mathrm{F}_{4}$ generation by the same way. Each treatment was performed for three replicates. The number of $D$. melanogaster of each generation was counted, and the male-to-female ratio was calculated for each generation to investigate the effect of V. coloratum polysaccharide on the fertility ${ }^{[20-21]}$.

\subsection{Determination of longevity}

The average and maximum lifespans were measured. Unmated female and male were collected for isolated culture. Referring to the method of Ye et al. ${ }^{[20-21]}$, male and female were inoculated with 10 bottles in the medium containing $50 \mathrm{mg} / \mathrm{L}$, $100 \mathrm{mg} / \mathrm{L}, 200 \mathrm{mg} / \mathrm{L}$ or $400 \mathrm{mg} / \mathrm{L}$, respectively. They were observed and recorded daily, and the number of dead individuals was recorded until the last $D$. melanogaster died. The data were analyzed to calculate the lifespans according to following equations. Average lifespan=sum of lifespans /total number of D. melanogaster; and maximum lifespan=sum of the survival time of the last four D. melanogaster that died/4. Briefly, individuals of $\mathrm{F}_{1}-\mathrm{F}_{4}$ generations were taken from those in fertility test for the determination of lifespan, and the method was performed as above. The differences in mortality were compared.

\subsection{Determination of survival time under acute injury}

The grouping way was the same as described in "Determination of fruit flies' longevity". A $20 \mathrm{mmol} / \mathrm{L}$ paraquat solution and a $9 \% \mathrm{H}_{2} \mathrm{O}_{2}$ solution were prepared with a $6 \%$ glucose solution for future use. The collected D. melanogaster (regardless of male and female) were isolated and starved for $2 \mathrm{~h}$, and then transferred to culture tubes. Next, $2 \mathrm{ml}$ of paraquat solution and $\mathrm{H}_{2} \mathrm{O}_{2}$ solution were dropped on a filter paper strip until the filter paper strip was complete wet, which was then placed in one of the culture tubes. The number of died $D$. melanogaster was observed every $4 \mathrm{~h}$ until all were died. The average survival time, half survival time and maximum survival time in each group were recorded (Li et al., 2007; Harman, 1981).

\subsection{Determination of antioxidant activity}

The method used in this test was performed as previous described with some modification ${ }^{[20]}$. First, 50 individuals cultured for $30 \mathrm{~d}$ were transferred to a blank culture tube containing wet filter paper, and they were anesthetized with diethyl ether. After weighing, they were put into a mortar that had been pre-cooled at $4{ }^{\circ} \mathrm{C}$, and then pre-cooled normal saline was added at a ratio of $49: 1(\mu \mathrm{l} / \mathrm{mg})$ for full grinding. The mixture was centrifuged at $3500 \mathrm{rpm} / \mathrm{min}$ for $15 \mathrm{~min}$. The supernatant was taken to further experiment. Superoxide dismutase (SOD) activity, catalase (CAT) activity and malondialdehyde (MDA) content in the supernatant were determined by SOD kit, CAT kit, MDA kit and protein kit. The reagents used in the determination must be prepared strictly in accordance with the instructions, and the determination methods must also strictly follow the conditions recommended by the manufacturer. 


\subsection{Statistical analysis}

All data were expressed as $\bar{x} \pm s$. SPSS 22.0 software (International Business Machines, corp., Armonk, NY, USA) was used for statistical analysis. The difference was performed by One-way analysis of variance (ANOVA) for the significance between control and treatment groups. $P<0.05$ indicated a significant difference and $P<0.01$ indicated an extremely significant difference.

\section{Results and Discussion}

\subsection{Characteristics of V. coloratum polysaccharide}

$V$. coloratum polysaccharide is a white powder obtained by enzymolysis with complex enzyme, followed by separation and extraction, decolorization and vacuum freeze-drying. It has been determined that the yield of $V$. coloratum polysaccharide was $19.83 \%$. The obtained $V$. coloratum polysaccharide was prepared into a solution with a concentration of $0.01 \%$, and its content was determined by UV spectrophotometry. It showed no characteristic absorption of proteins and nucleic acids at 260 and $280 \mathrm{~nm}$, respectively. However, one crack-free peak appeared by using HPLC-GPC, indicating that $V$. coloratum polysaccharide was a kind of polysaccharide, and its purity was $99.95 \%$.

\subsection{Effects of V. coloratum polysaccharide on fertility of D. melanogaster}

The results of different concentration of $V$. coloratum polysaccharide on the fertility, total number and male-to-female ratio of $\mathrm{F} 1$ to $\mathrm{F} 4$ generations of $D$. melanogaster were shown in Table 1- 3. With the increased concentration, the number of female and male in the $\mathrm{F}_{1}-\mathrm{F}_{4}$ generations were all increased. When the concentration was $50 \mathrm{mg} / \mathrm{L}$, both the number of female and male in each of the $\mathrm{F}_{1}-\mathrm{F}_{4}$ generations and the total number of all generations were significantly higher than those in control group $(P<0.01)$. When the concentration was $400 \mathrm{mg} / \mathrm{L}$, both the number of female and male in each of the $\mathrm{F}_{1}-\mathrm{F}_{4}$ generations and the total number of all generations were $200 \%$ of those in control group. When the concentration was between 50 and $400 \mathrm{mg} / \mathrm{L}$, the male-to-female sex ratio did not change significantly in the $\mathrm{F}_{1}-\mathrm{F}_{4}$ generations, and the overall ratio was close to 1:1. Therefore, the above results suggested that $V$. coloratum polysaccharide had a significant promotion effect on the fertility of fruit flies.

Table 1. Effects of V. coloratum polysaccharide in different concentrations on generational fertility of D. melanogaster $(\bar{x} \pm s$, flies).

\begin{tabular}{|c|c|c|c|c|c|c|c|c|}
\hline \multirow{2}{*}{$\begin{array}{c}\text { Polysaccharide } \\
\text { concentration }(\mathrm{mg} / \mathrm{L})\end{array}$} & \multicolumn{2}{|c|}{$\mathrm{F}_{1}$} & \multicolumn{2}{|c|}{$\mathrm{F}_{2}$} & \multicolumn{2}{|c|}{$\mathrm{F}_{3}$} & \multicolumn{2}{|c|}{$\mathrm{F}_{4}$} \\
\hline & q & $\hat{0}$ & 우 & $\hat{0}$ & 우 & $\hat{0}$ & 우 & $\hat{\sigma}$ \\
\hline CK $(0)$ & $298 \pm 3.13^{\mathrm{aA}}$ & $299 \pm 2.62^{\mathrm{aA}}$ & $274 \pm 1.53^{\mathrm{aA}}$ & $270 \pm 1.72^{\mathrm{aA}}$ & $244 \pm 1.92^{\mathrm{aA}}$ & $233 \pm 0.62^{\mathrm{aA}}$ & $235 \pm 3.13^{\mathrm{aA}}$ & $228 \pm 2.62^{\mathrm{aA}}$ \\
\hline 100 & $331 \pm 2.61^{\mathrm{bA}}$ & $334 \pm 2.65^{\mathrm{bA}}$ & $324 \pm 1.47^{\mathrm{bA}}$ & $314 \pm 1.55^{\mathrm{bA}}$ & $302 \pm 1.53^{\mathrm{bB}}$ & $296 \pm 1.68^{\mathrm{bB}}$ & $296 \pm 2.61^{\mathrm{bB}}$ & $293 \pm 2.65^{\mathrm{bB}}$ \\
\hline 200 & $437 \pm 2.44^{\mathrm{cB}}$ & $440 \pm 2.28^{\mathrm{cB}}$ & $431 \pm 1.65^{\mathrm{cB}}$ & $428 \pm 1.37^{\mathrm{cB}}$ & $407 \pm 2.43^{\mathrm{cC}}$ & $402 \pm 0.53^{\mathrm{cC}}$ & $409 \pm 2.44^{\mathrm{cC}}$ & $404 \pm 2.28^{\mathrm{cC}}$ \\
\hline
\end{tabular}

Different lowercase letters represent a significant difference $(P<0.05)$, and uppercase letters stand for an extremely significant difference $(P<0.01)$.

Table 2. Effects of $V$. coloratum polysaccharide in different concentrations on total number in different generations of $D$. melanogaster $(\bar{x} \pm s$, flies).

\begin{tabular}{|c|c|c|c|c|c|c|c|}
\hline \multirow{2}{*}{$\begin{array}{c}\text { Polysaccharide } \\
\text { concentration }(\mathrm{mg} / \mathrm{L})\end{array}$} & \multirow{2}{*}{$\mathrm{F}_{1}$} & \multirow{2}{*}{$\mathrm{F}_{2}$} & \multirow{2}{*}{$\mathrm{F}_{3}$} & \multirow{2}{*}{$\mathrm{F}_{4}$} & \multicolumn{3}{|c|}{ Total } \\
\hline & & & & & 운 & $0^{1}$ & $q+0^{\lambda}$ \\
\hline $\mathrm{CK}(0)$ & $597 \pm 3.70^{\mathrm{aA}}$ & $544 \pm 3.63^{\mathrm{aA}}$ & $477 \pm 3.21^{\mathrm{aA}}$ & $463 \pm 3.67^{\mathrm{aA}}$ & $1051 \pm 3.27^{\mathrm{aA}}$ & $1030 \pm 3.37^{\mathrm{aA}}$ & $2081 \pm 7.80^{\mathrm{aA}}$ \\
\hline 50 & $625 \pm 3.21^{\mathrm{bA}}$ & $583 \pm 3.01^{\mathrm{bA}}$ & $412 \pm 3.62^{\mathrm{bA}}$ & $495 \pm 3.61^{\mathrm{bA}}$ & $1114 \pm 3.67^{\mathrm{bB}}$ & $1101 \pm 4.77$ ьв & $2215 \pm 7.58^{\mathrm{bB}}$ \\
\hline 100 & $665 \pm 3.61^{\mathrm{cB}}$ & $638 \pm 3.19^{\mathrm{cB}}$ & $598 \pm 3.57^{\mathrm{cB}}$ & $589 \pm 3.65^{\mathrm{cB}}$ & $1253 \pm 5.08^{\mathrm{cB}}$ & $1237 \pm 5.21^{\mathrm{cC}}$ & $2490 \pm 7.81^{\mathrm{cC}}$ \\
\hline 200 & $877 \pm 2.80^{\mathrm{dC}}$ & $859 \pm 2.60^{\mathrm{dC}}$ & $809 \pm 3.50^{\mathrm{dC}}$ & $813 \pm 3.62^{\mathrm{dC}}$ & $1684 \pm 4.50^{\mathrm{dC}}$ & $1674 \pm 4.54^{\mathrm{dD}}$ & $3358 \pm 8.50^{\mathrm{dD}}$ \\
\hline 400 & $978 \pm 2.97^{\mathrm{eD}}$ & $955 \pm 3.20^{\mathrm{eD}}$ & $988 \pm 2.99 \mathrm{eD}$ & $951 \pm 2.81^{\mathrm{eD}}$ & $1938 \pm 2.84^{\mathrm{eD}}$ & $1934 \pm 4.81^{\mathrm{eE}}$ & $3872 \pm 7.70^{\mathrm{eE}}$ \\
\hline
\end{tabular}

Different lowercase letters represent a significant difference $(P<0.05)$, and uppercase letters stand for an extremely significant difference $(P<0.01)$.

Table 3. Effects of V. coloratum polysaccharide in different concentrations on sex ratio in different generations of D. melanogaster.

\begin{tabular}{cccccc}
\hline $\begin{array}{c}\text { Polysaccharide } \\
\text { concentration }(\mathrm{mg} / \mathrm{L})\end{array}$ & $\mathrm{F}_{1}$ & $\mathrm{~F}_{2}$ & $\mathrm{~F}_{3}$ & $\mathrm{~F}_{4}$ & Mean \\
\hline CK $(0)$ & 0.997 & 1.014 & 1.047 & 1.030 & 1.029 \\
50 & 0.978 & 1.024 & 1.024 & 1.010 & 1.014 \\
100 & 0.991 & 1.031 & 1.020 & 1.012 & 1.013 \\
200 & 0.993 & 1.007 & 1.012 & 0.994 & 1.006 \\
400 & 0.992 & 1.011 & 1.012 & & \\
\hline
\end{tabular}




\subsection{Effects of V. coloratum polysaccharide on longevity of D. melanogaster}

Table 4 and Table 5 showed the results of different concentrations of $V$. coloratum polysaccharide on the generational lifespan and accumulated lifespan of $D$. melanogaster, respectively. With the increased concentration, the lifespans of female and male in the $\mathrm{F}_{1}-\mathrm{F}_{4}$ generations both increased. When the concentration was $50 \mathrm{mg} / \mathrm{L}$, the accumulated lifespans of female and male, the total lifespan of female and male and the longest lifespans of female and male in the $\mathrm{F}_{1}-\mathrm{F}_{4}$ generation were significantly increased than those in control group, respectively $(P<0.05)$. When the concentration was 100,200 and $400 \mathrm{mg} / \mathrm{L}$, the accumulated lifespans, the total lifespan and the longest lifespans in the $\mathrm{F}_{1}-\mathrm{F}_{4}$ generations were extremely significant increase than those in control group $(P<0.01)$. Those results showed that $V$. coloratum polysaccharide had a significant effect on prolonging the lifespans of D. melanogaster.

\subsection{Effects of V. coloratum polysaccharide on survival time of D. melanogaster under acute injury}

Table 6 and Table 7 showed the effects of different concentrations of $V$. coloratum polysaccharide on the survival time of D. melanogaster under acute injury. When treated with hydrogen peroxide and paraquat, the survival time in the $\mathrm{F}_{1}-\mathrm{F}_{4}$ generations was significantly prolonged after intervention with 200-400 $\mathrm{mg} / \mathrm{L}$ V. coloratum polysaccharide in comparison with the control group $(P<0.05)$. When the concentration was $400 \mathrm{mg} / \mathrm{L}$, the average survival time in the $\mathrm{F}_{1}-\mathrm{F}_{4}$ generations treated with hydrogen peroxide and paraquat were increased by about $6 \mathrm{~h}$ in comparison with the control group, the half survival time were also increased by about $4 \mathrm{~h}$ in comparison with the control group, and the longest survival time were increased by about $5 \mathrm{~h}$ in comparison with the control group. The above results showed that $V$. coloratum polysaccharide had significant effects on prolonging the survival time of $D$. melanogaster with acute injury.

Table 4. Effects of $V$. coloratum polysaccharide in different concentrations on generational lifespan of D. melanogaster $(\bar{x} \pm s, \mathrm{~d})$.

\begin{tabular}{|c|c|c|c|c|c|c|c|c|}
\hline \multirow{2}{*}{$\begin{array}{c}\text { Polysaccharide } \\
\text { concentration }(\mathrm{mg} / \mathrm{L})\end{array}$} & \multicolumn{2}{|c|}{ F1 } & \multicolumn{2}{|c|}{ F2 } & \multicolumn{2}{|c|}{ F3 } & \multicolumn{2}{|c|}{ F4 } \\
\hline & q & $\hat{0}$ & q & $\hat{\jmath}$ & q & $\hat{0}$ & 우 & $\hat{0}$ \\
\hline CK $(0)$ & $50.89 \pm 0.36^{\mathrm{aA}}$ & $51.61 \pm 0.46^{\mathrm{aA}}$ & $56.94 \pm 0.44^{\text {aA }}$ & $51.82 \pm 0.26^{\mathrm{aA}}$ & $54.79 \pm 0.26^{\mathrm{aA}}$ & $54.90 \pm 0.70^{\mathrm{aA}}$ & $50.54 \pm 0.64^{\mathrm{aA}}$ & $54.65 \pm 0.94^{\mathrm{aA}}$ \\
\hline 50 & $51.88 \pm 0.58^{\mathrm{aA}}$ & $53.92 \pm 0.30^{\mathrm{aA}}$ & $57.62 \pm 0.72^{\mathrm{aA}}$ & $54.54 \pm 0.28^{\mathrm{aA}}$ & $56.81 \pm 0.35^{\mathrm{aA}}$ & $52.10 \pm 0.34^{\mathrm{aA}}$ & $53.64 \pm 0.32^{\mathrm{aA}}$ & $59.90 \pm 0.43^{\mathrm{aA}}$ \\
\hline 200 & $74.63 \pm 0.40^{\text {св }}$ & $76.04 \pm 0.48^{\mathrm{cB}}$ & $78.01 \pm 0.72^{\text {св }}$ & $78.85 \pm 0.70^{\text {св }}$ & $73.94 \pm 0.19^{\mathrm{cB}}$ & $70.95 \pm 0.32^{\mathrm{cB}}$ & $73.84 \pm 0.41^{\mathrm{cB}}$ & $78.96 \pm 0.32^{\text {сВ }}$ \\
\hline 400 & $79.73 \pm 0.72^{\mathrm{dC}}$ & $78.98 \pm 0.52^{\mathrm{dC}}$ & $81.01 \pm 0.32^{\mathrm{dC}}$ & $81.88 \pm 1.00^{\mathrm{dC}}$ & $82.91 \pm 0.30^{\mathrm{dC}}$ & $79.11 \pm 0.32^{\mathrm{dC}}$ & $80.18 \pm 0.43^{\mathrm{dC}}$ & $83.24 \pm 0.63^{\mathrm{dC}}$ \\
\hline
\end{tabular}

Different lowercase letters represent a significant difference $(P<0.05)$, and uppercase letters stand for an extremely significant difference $(P<0.01)$.

Table 5. Effects of $V$. coloratum polysaccharide in different concentrations on accumulated lifespan of fruit fly generations ( $\bar{x} \pm s, \mathrm{~d})$.

\begin{tabular}{|c|c|c|c|c|c|c|}
\hline \multirow{2}{*}{$\begin{array}{c}\text { Polysaccharide } \\
\text { concentration }(\mathrm{mg} / \mathrm{L})\end{array}$} & \multicolumn{2}{|c|}{ Accumulated lifespan of $\left(\mathrm{F}_{1}-\mathrm{F}_{4}\right)$} & \multicolumn{2}{|c|}{ Average lifespan of $\left(\mathrm{F}_{1}-\mathrm{F}_{4}\right)$} & \multicolumn{2}{|c|}{ The longest lifespan of $\left(\mathrm{F}_{1}-\mathrm{F}_{4}\right)$} \\
\hline & 우 & $\hat{\sigma}$ & q & o & $q$ & $\hat{0}$ \\
\hline $\mathrm{CK}(0)$ & $213.16 \pm 4.11^{\mathrm{aA}}$ & $213.01 \pm 4.79^{\mathrm{aA}}$ & $53.29 \pm 0.72^{\mathrm{aA}}$ & $53.25 \pm 0.65^{\mathrm{aA}}$ & $57.81 \pm 0.55^{\mathrm{aA}}$ & $58.74 \pm 0.39^{\mathrm{aA}}$ \\
\hline 100 & $277.54 \pm 3.09^{\mathrm{cB}}$ & $265.38 \pm 2.82^{\mathrm{cB}}$ & $69.39 \pm 0.91^{\mathrm{bB}}$ & $66.35 \pm 0.79^{\mathrm{bB}}$ & $72.80 \pm 0.49^{c \mathrm{C}}$ & $73.73 \pm 0.43^{\mathrm{cC}}$ \\
\hline 200 & $300.42 \pm 2.45^{\mathrm{dC}}$ & $304.80 \pm 0.75^{\mathrm{dC}}$ & $75.11 \pm 0.67^{\mathrm{cC}}$ & $76.20 \pm 0.50 \mathrm{cC}$ & $80.72 \pm 0.76^{\mathrm{dD}}$ & $77.39 \pm 0.47^{\mathrm{dD}}$ \\
\hline
\end{tabular}

Different lowercase letters represent a significant difference $(P<0.05)$, and uppercase letters stand for an extremely significant difference $(P<0.01)$.

Table 6. Effects of V. coloratum polysaccharide in different concentrations on the survival time in paraquat treatment of $D$. melanogaster $(\bar{x} \pm s, \mathrm{~h})$.

\begin{tabular}{|c|c|c|c|c|c|c|c|c|c|c|c|c|}
\hline \multirow{2}{*}{$\begin{array}{l}\text { Polysaccharide } \\
\text { concentration } \\
(\mathrm{mg} / \mathrm{L})\end{array}$} & \multicolumn{3}{|c|}{$\mathrm{F}_{1}$} & \multicolumn{3}{|c|}{$\mathrm{F}_{1}$} & \multicolumn{3}{|c|}{$\mathrm{F}_{3}$} & \multicolumn{3}{|c|}{$\mathrm{F}_{4}$} \\
\hline & $\begin{array}{c}\text { Average } \\
\text { survival } \\
\text { time }\end{array}$ & $\begin{array}{l}\text { Half survival } \\
\text { time }\end{array}$ & $\begin{array}{l}\text { The longest } \\
\text { survival } \\
\text { time }\end{array}$ & $\begin{array}{l}\text { Average } \\
\text { survival } \\
\text { time }\end{array}$ & $\begin{array}{l}\text { Half survival } \\
\text { time }\end{array}$ & $\begin{array}{l}\text { The longest } \\
\text { survival } \\
\text { time }\end{array}$ & $\begin{array}{l}\text { Average } \\
\text { survival } \\
\text { time }\end{array}$ & $\begin{array}{l}\text { Half survival } \\
\text { time }\end{array}$ & $\begin{array}{l}\text { The longest } \\
\text { survival } \\
\text { time }\end{array}$ & $\begin{array}{l}\text { Average } \\
\text { survival } \\
\text { time }\end{array}$ & $\begin{array}{l}\text { Half survival } \\
\text { time }\end{array}$ & $\begin{array}{c}\text { The longest } \\
\text { survival } \\
\text { time }\end{array}$ \\
\hline CK $(0)$ & $25.12 \pm 1.04^{\mathrm{aA}}$ & $23.12 \pm 0.84^{\mathrm{aA}}$ & $38.17 \pm 0.76^{\mathrm{aA}}$ & $24.92 \pm 1.14^{\mathrm{aA}}$ & $23.39 \pm 0.54^{\mathrm{aA}}$ & $38.49 \pm 0.96^{\mathrm{aA}}$ & $25.32 \pm 1.64^{\mathrm{aA}}$ & $23.86 \pm 0.77^{\mathrm{aA}}$ & $38.21 \pm 0.19^{\mathrm{aA}}$ & $25.41 \pm 1.09^{\mathrm{aA}}$ & $23.36 \pm 0.97^{\mathrm{aA}}$ & $38.11 \pm 0.14^{\mathrm{aA}}$ \\
\hline 50 & $27.44 \pm 1.76^{\mathrm{aA}}$ & $24.54 \pm 0.91^{\mathrm{aA}}$ & $39.32 \pm 0.59^{\mathrm{aA}}$ & $27.64 \pm 0.26^{\mathrm{bB}}$ & $24.94 \pm 0.31^{\mathrm{aA}}$ & $39.92 \pm 0.99^{\mathrm{aA}}$ & $27.86 \pm 0.39^{\mathrm{bB}}$ & $25.04 \pm 0.86^{\mathrm{aA}}$ & $40.03 \pm 0.91^{\mathrm{aA}}$ & $28.16 \pm 0.77^{\mathrm{bB}}$ & $25.64 \pm 0.38^{\mathrm{aA}}$ & $40.63 \pm 0.29^{\mathrm{aA}}$ \\
\hline 100 & $28.28 \pm 1.74^{\mathrm{bA}}$ & $25.25 \pm 0.24^{\mathrm{bA}}$ & $39.36 \pm 0.66^{\mathrm{aA}}$ & $28.78 \pm 0.14^{\mathrm{bB}}$ & $25.55 \pm 0.26^{\mathrm{bA}}$ & $40.56 \pm 0.96^{\mathrm{bA}}$ & $29.15 \pm 0.63^{\mathrm{cB}}$ & $25.63 \pm 0.56^{\mathrm{aA}}$ & $40.96 \pm 0.66^{\mathrm{bA}}$ & $29.75 \pm 0.58^{\mathrm{bB}}$ & $25.83 \pm 0.57^{\mathrm{aA}}$ & $41.69 \pm 0.87^{\mathrm{bA}}$ \\
\hline 200 & $30.25 \pm 1.07^{\mathrm{CA}}$ & $26.18 \pm 0.55^{\mathrm{bA}}$ & $40.25 \pm 1.09^{\mathrm{bA}}$ & $30.65 \pm 0.27^{\mathrm{cB}}$ & $26.54 \pm 0.39^{\mathrm{bA}}$ & $40.95 \pm 0.89^{\mathrm{bA}}$ & $31.55 \pm 0.87^{\mathrm{dC}}$ & $26.89 \pm 0.46^{\mathrm{bA}}$ & $41.15 \pm 0.94^{\mathrm{bA}}$ & $31.65 \pm 0.83^{\mathrm{CC}}$ & $27.19 \pm 0.34^{\mathrm{bA}}$ & $41.65 \pm 0.44^{\mathrm{bA}}$ \\
\hline 400 & $31.12 \pm 0.86^{\mathrm{CA}}$ & $27.39 \pm 0.38^{\mathrm{cA}}$ & $40.86 \pm 0.96^{\mathrm{bA}}$ & $31.29 \pm 0.39^{\mathrm{cB}}$ & $27.61 \pm 0.68^{\mathrm{CB}}$ & $41.69 \pm 0.76^{\mathrm{cB}}$ & $31.59 \pm 0.59^{\mathrm{dC}}$ & $28.32 \pm 0.49^{\mathrm{cB}}$ & $41.85 \pm 0.51^{\mathrm{CB}}$ & $31.87 \pm 0.39^{\circ C}$ & $28.82 \pm 0.89^{\mathrm{CB}}$ & $42.15 \pm 0.91^{\mathrm{cB}}$ \\
\hline
\end{tabular}

Different lowercase letters represent a significant difference $(P<0.05)$, and uppercase letters stand for an extremely significant difference $(P<0.01)$. 
Table 7. Effects of $V$. coloratum polysaccharide in different concentrations on the survival time in hydrogen peroxide treatment of $D . m e l a n o g a s t e r(\bar{x} \pm s, \mathrm{~h})$.

\begin{tabular}{|c|c|c|c|c|c|c|c|c|c|c|c|c|}
\hline \multirow{2}{*}{$\begin{array}{c}\text { Polysaccharide } \\
\text { concentration } \\
(\mathrm{mg} / \mathrm{L})\end{array}$} & \multicolumn{3}{|c|}{$\mathrm{F}_{1}$} & \multicolumn{3}{|c|}{$\mathrm{F}_{2}$} & \multicolumn{3}{|c|}{$\mathrm{F}_{3}$} & \multicolumn{3}{|c|}{$\mathrm{F}_{4}$} \\
\hline & $\begin{array}{c}\text { Average } \\
\text { survival } \\
\text { time }\end{array}$ & $\begin{array}{l}\text { Half survival } \\
\text { time }\end{array}$ & $\begin{array}{c}\text { The longest } \\
\text { survival } \\
\text { time }\end{array}$ & $\begin{array}{c}\text { Average } \\
\text { survival } \\
\text { time }\end{array}$ & $\begin{array}{l}\text { Half survival } \\
\text { time }\end{array}$ & $\begin{array}{c}\text { The longest } \\
\text { survival } \\
\text { time }\end{array}$ & $\begin{array}{c}\text { Average } \\
\text { survival } \\
\text { time }\end{array}$ & $\begin{array}{l}\text { Half survival } \\
\text { time }\end{array}$ & $\begin{array}{c}\text { The longest } \\
\text { survival } \\
\text { time }\end{array}$ & $\begin{array}{c}\text { Average } \\
\text { survival } \\
\text { time }\end{array}$ & $\begin{array}{l}\text { Half survival } \\
\text { time }\end{array}$ & $\begin{array}{c}\text { The longest } \\
\text { survival } \\
\text { time }\end{array}$ \\
\hline $\mathrm{CK}(0)$ & $26.00 \pm 1.07^{\mathrm{aA}}$ & $23.89 \pm 0.96^{\mathrm{aA}}$ & $38.48 \pm 0.87^{\mathrm{aA}}$ & $25.19 \pm 1.26^{\mathrm{aA}}$ & $23.69 \pm 0.65^{\mathrm{aA}}$ & $38.79 \pm 0.36^{\mathrm{aA}}$ & $25.64 \pm 1.04^{\mathrm{aA}}$ & $24.03 \pm 0.64^{\mathrm{aA}}$ & $38.36 \pm 0.39^{\mathrm{aA}}$ & $25.56 \pm 0.69^{\mathrm{aA}}$ & $23.86 \pm 0.67^{\mathrm{aA}}$ & $38.61 \pm 0.74^{\mathrm{aA}}$ \\
\hline 50 & $29.32 \pm 1.79^{\mathrm{aA}}$ & $25.16 \pm 1.01^{\mathrm{aA}}$ & $39.63 \pm 0.70^{\text {aA }}$ & $28.01 \pm 0.37^{\mathrm{bB}}$ & $25.24 \pm 0.45^{\mathrm{aA}}$ & $40.22 \pm 0.39^{\mathrm{aA}}$ & $28.16 \pm 0.33^{\mathrm{bB}}$ & $25.22 \pm 0.56^{\mathrm{AA}}$ & $40.25 \pm 0.84^{\mathrm{aA}}$ & $28.33 \pm 0.67^{\mathrm{bB}}$ & $26.13 \pm 0.38^{\mathrm{aA}}$ & $41.13 \pm 0.89^{\mathrm{aA}}$ \\
\hline 100 & $29.16 \pm 1.56^{\mathrm{bA}}$ & $25.99 \pm 0.36^{\mathrm{bA}}$ & $39.67 \pm 0.77^{\mathrm{aA}}$ & $29.15 \pm 0.28^{\text {bв }}$ & $25.83 \pm 0.35^{\mathrm{bA}}$ & $40.87 \pm 0.84^{\mathrm{bA}}$ & $29.48 \pm 0.74^{\mathrm{cB}}$ & $25.80 \pm 0.44^{\mathrm{aA}}$ & $41.14 \pm 0.96^{\mathrm{bA}}$ & $29.95 \pm 0.48^{\mathrm{bB}}$ & $26.33 \pm 0.52^{\mathrm{aA}}$ & $42.19 \pm 0.67^{\mathrm{bA}}$ \\
\hline 200 & $31.13 \pm 1.07^{\mathrm{cA}}$ & $26.90 \pm 0.67^{\mathrm{bA}}$ & $40.59 \pm 1.01^{\mathrm{bA}}$ & $30.92 \pm 0.47^{\mathrm{cB}}$ & $26.86 \pm 0.62^{\mathrm{bA}}$ & $41.25 \pm 0.19^{\mathrm{bA}}$ & $31.88 \pm 0.39^{\mathrm{dC}}$ & $27.06 \pm 0.18^{\mathrm{bA}}$ & $41.65 \pm 0.64^{\mathrm{bA}}$ & $31.85 \pm 0.33^{c \mathrm{C}}$ & $27.69 \pm 0.64^{\mathrm{bA}}$ & $42.35 \pm 0.49^{\mathrm{bA}}$ \\
\hline 400 & $32.02 \pm 0.86^{\mathrm{cA}}$ & $28.09 \pm 0.38^{\mathrm{cA}}$ & $41.16 \pm 0.86^{\mathrm{bA}}$ & $31.57 \pm 0.52^{\mathrm{cB}}$ & $27.94 \pm 0.61^{\mathrm{cB}}$ & $41.91 \pm 0.71^{\mathrm{cB}}$ & $31.96 \pm 0.94^{\mathrm{dC}}$ & $28.58 \pm 0.99^{\mathrm{cB}}$ & $42.35 \pm 0.81^{c \mathrm{~B}}$ & $32.07 \pm 0.69^{c C}$ & $29.32 \pm 0.69^{\mathrm{cB}}$ & $43.95 \pm 1.11^{\mathrm{cB}}$ \\
\hline
\end{tabular}

Different lowercase letters represent a significant difference $(P<0.05)$, and uppercase letters stand for an extremely significant difference $(P<0.01)$.

Table 8. Effects of V. coloratum polysaccharide in different concentrations on superoxide dismutase activity of D. melanogaster $(\bar{x} \pm s, \mathrm{U} / \mathrm{mg} \bullet \operatorname{protein})$.

\begin{tabular}{|c|c|c|c|c|c|c|c|c|}
\hline \multirow{2}{*}{$\begin{array}{c}\text { Polysaccharide } \\
\text { concentration }(\mathrm{mg} / \mathrm{L})\end{array}$} & \multicolumn{2}{|c|}{$\mathrm{F}_{1}$} & \multicolumn{2}{|c|}{$\mathrm{F}_{2}$} & \multicolumn{2}{|c|}{$\mathrm{F}_{3}$} & \multicolumn{2}{|c|}{$\mathrm{F}_{4}$} \\
\hline & q & 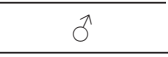 & $q$ & $\delta$ & q & 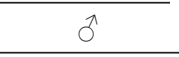 & $q$ & $\pi$ \\
\hline $\mathrm{CK}(0)$ & $31.42 \pm 0.34^{\mathrm{bA}}$ & $35.92 \pm 0.41^{\mathrm{bA}}$ & $32.40 \pm 0.38^{\mathrm{bA}}$ & $35.90 \pm 0.45^{\mathrm{bA}}$ & $30.91 \pm 0.70^{\mathrm{bA}}$ & $35.31 \pm 0.67^{\mathrm{bA}}$ & $31.20 \pm 0.80^{\mathrm{bA}}$ & $34.75 \pm 0.74^{\mathrm{bA}}$ \\
\hline 100 & $36.51 \pm 0.47^{\mathrm{cB}}$ & $40.01 \pm 0.54^{\mathrm{cB}}$ & $38.73 \pm 0.51^{\mathrm{cB}}$ & $42.23 \pm 0.62^{\mathrm{cB}}$ & $37.41 \pm 0.58^{\mathrm{cB}}$ & $40.93 \pm 0.50^{\text {св }}$ & $37.30 \pm 0.56^{\mathrm{cB}}$ & $40.63 \pm 0.44^{\mathrm{cB}}$ \\
\hline 200 & $41.28 \pm 0.92^{\mathrm{dB}}$ & $44.78 \pm 0.81^{\mathrm{dB}}$ & $43.20 \pm 0.40^{\mathrm{dB}}$ & $47.73 \pm 0.58^{\mathrm{dB}}$ & $43.31 \pm 0.80^{\mathrm{dB}}$ & $47.78 \pm 0.86^{\mathrm{dB}}$ & $45.25 \pm 0.38^{\mathrm{dB}}$ & $48.47 \pm 0.91^{\mathrm{dB}}$ \\
\hline
\end{tabular}

Different lowercase letters represent a significant difference $(P<0.05)$, and uppercase letters stand for an extremely significant difference $(P<0.01)$.

Table 9. Effects of $V$. coloratum polysaccharide in different concentrations on catalase activity of D. melanogaster $(\bar{x} \pm s, \mathrm{U} / \mathrm{mg} \bullet$ protein $)$.

\begin{tabular}{|c|c|c|c|c|c|c|c|c|}
\hline \multirow{2}{*}{$\begin{array}{c}\text { Polysaccharide } \\
\text { concentration }(\mathrm{mg} / \mathrm{L})\end{array}$} & \multicolumn{2}{|c|}{$\mathrm{F}_{1}$} & \multicolumn{2}{|c|}{$\mathrm{F}_{2}$} & \multicolumn{2}{|c|}{$\mathrm{F}_{3}$} & \multicolumn{2}{|c|}{$\mathrm{F}_{4}$} \\
\hline & 운 & 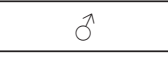 & $q$ & $\hat{0}$ & q & $\hat{0}$ & q & $\hat{0}$ \\
\hline $\mathrm{CK}(0)$ & $7.73 \pm 0.32^{\mathrm{aA}}$ & $8.61 \pm 0.89^{\mathrm{aA}}$ & $7.61 \pm 0.47^{\mathrm{aA}}$ & $7.83 \pm 0.68^{\mathrm{aA}}$ & $7.65 \pm 0.75^{\mathrm{aA}}$ & $8.70 \pm 0.91^{\mathrm{aA}}$ & $8.70 \pm 0.96^{\mathrm{aA}}$ & $10.00 \pm 1.12^{\mathrm{aA}}$ \\
\hline 100 & $10.17 \pm 0.42^{\mathrm{bA}}$ & $11.41 \pm 0.35^{\mathrm{bA}}$ & $10.67 \pm 0.52^{\mathrm{bA}}$ & $11.91 \pm 0.66^{\mathrm{bA}}$ & $10.15 \pm 0.55^{\mathrm{bA}}$ & $11.45 \pm 1.17^{\mathrm{bA}}$ & $10.54 \pm 0.86^{\mathrm{bA}}$ & $11.90 \pm 1.01^{\mathrm{bA}}$ \\
\hline 200 & $11.27 \pm 0.56^{\mathrm{bA}}$ & $12.51 \pm 0.60^{\mathrm{bA}}$ & $11.37 \pm 0.87^{\mathrm{bA}}$ & $12.59 \pm 0.95^{\mathrm{bA}}$ & $11.38 \pm 0.86^{\mathrm{bA}}$ & $12.62 \pm 0.89^{\mathrm{bA}}$ & $11.16 \pm 0.41^{\mathrm{bA}}$ & $12.56 \pm 0.73^{\mathrm{bA}}$ \\
\hline
\end{tabular}

Different lowercase letters represent a significant difference $(P<0.05)$, and uppercase letters stand for an extremely significant difference $(P<0.01)$.

\subsection{Effects of different concentrations of $V$. coloratum polysaccharide on anti-oxidase activity and malondialdehyde content of D. melanogaster}

With the increased concentration of $V$. coloratum polysaccharide, the SOD activity in male and female in the $\mathrm{F}_{1}-\mathrm{F}_{4}$ generations showed a trend of first decreasing and then increasing (Table 8). When the concentration was $50 \mathrm{mg} / \mathrm{L}$, the SOD activity in the $\mathrm{F}_{1}-\mathrm{F}_{4}$ generations was the lowest $(P<0.05)$. When the concentrations were gradually increased from 100 to $400 \mathrm{mg} / \mathrm{L}$, the SOD activity in the $\mathrm{F}_{1}-\mathrm{F}_{4}$ generations gradually increased $(P<0.05, P<0.01)$.

Moreover, with the increase of concentration, the CAT activity in the $\mathrm{F}_{1}-\mathrm{F}_{4}$ generations showed an increased trend (Table 9). When the concentration was $50 \mathrm{mg} / \mathrm{L}$, the CAT activity in the $\mathrm{F}_{1}-\mathrm{F}_{4}$ generations was significantly increased than those in control group $(P<0.05)$. When the concentration were gradually increased from 100 to $400 \mathrm{mg} / \mathrm{L}$, the CAT activity in the $\mathrm{F}_{1}-\mathrm{F}_{4}$ generations were extremely significant increased than those in control group $(P<0.01)$.

Furthermore, with the increase of concentration, the MDA contents in the $\mathrm{F}_{1}-\mathrm{F}_{4}$ generations all showed a trend of increasing first and then decreasing (Table 10). When the concentration was $50 \mathrm{mg} / \mathrm{L}$, the MDA contents in the $\mathrm{F}_{1}-\mathrm{F}_{4}$ generations were lower than those in control group, and when it was higher than $400 \mathrm{mg} / \mathrm{L}$, there were extremely significant differences from the control group $(P<0.01)$. When the concentration was $500 \mathrm{mg} / \mathrm{L}$, the MDA contents in $\mathrm{t}$ the $\mathrm{F}_{1}-\mathrm{F}_{4}$ generations were the lowest. It indicated that with the increased concentration, the effect of the $V$. coloratum polysaccharide on scavenging MDA was gradually enhanced, and the antioxidant effect was also gradually enhanced. The results showed that high dose of $V$. coloratum polysaccharide significantly increased the activity of SOD and 
Table 10. Effects of $V$. coloratum polysaccharide in different concentrations on malondialdehyde content of $D . m e l a n o g a s t e r(\bar{x} \pm s, \mathrm{nmol} / \mathrm{mg})$.

\begin{tabular}{|c|c|c|c|c|c|c|c|c|}
\hline $\begin{array}{c}\text { Polysaccharide } \\
\text { concentration }(\mathrm{mg} / \mathrm{L})\end{array}$ & \multicolumn{2}{|c|}{$\mathrm{F}_{1}$} & \multicolumn{2}{|c|}{$\mathrm{F}_{2}$} & \multicolumn{2}{|c|}{$\mathrm{F}_{2}$} & \multicolumn{2}{|c|}{$\mathrm{F}_{3}$} \\
\hline CK (0) & $1.18 \pm 0.06^{\mathrm{dB}}$ & $1.36 \pm 0.03^{\mathrm{dC}}$ & $1.17 \pm 0.05^{\mathrm{cB}}$ & $1.35 \pm 0.04^{\mathrm{dC}}$ & $1.20 \pm 0.04^{\mathrm{dC}}$ & $1.45 \pm 0.04^{\mathrm{dC}}$ & $1.44 \pm 0.03^{\mathrm{dC}}$ & $1.71 \pm 0.06^{\mathrm{dC}}$ \\
\hline 100 & $1.13 \pm 0.03^{\mathrm{cB}}$ & $1.21 \pm 0.03^{\mathrm{cB}}$ & $1.16 \pm 0.09^{\mathrm{cB}}$ & $1.19 \pm 0.07^{\mathrm{cB}}$ & $1.02 \pm 0.06^{\mathrm{cA}}$ & $1.20 \pm 0.04^{\mathrm{cA}}$ & $0.99 \pm 0.07^{\mathrm{cA}}$ & $1.17 \pm 0.06^{\mathrm{cB}}$ \\
\hline 200 & $0.94 \pm 0.08^{\mathrm{bA}}$ & $1.02 \pm 0.06^{\mathrm{bA}}$ & $0.99 \pm 0.05^{\mathrm{bA}}$ & $1.05 \pm 0.05^{\mathrm{bA}}$ & $0.91 \pm 0.07^{\mathrm{bA}}$ & $1.11 \pm 0.09^{\mathrm{bA}}$ & $0.84 \pm 0.05^{\mathrm{bA}}$ & $1.02 \pm 0.07^{\mathrm{bA}}$ \\
\hline
\end{tabular}

CAT and reduced the content of MDA in D. melanogaster in the $\mathrm{F}_{1}-\mathrm{F}_{4}$ generations, and improved the antioxidant capacity and vitality. It was also found that the activities of SOD and CAT and the content of MDA in female were lower than those in male with different concentrations of $V$. coloratum polysaccharide, which also showed that the antioxidant effect of female was stronger than that of male.

In this study, we explored the effects of $V$. coloratum polysaccharide on the fertility, longevity and antioxidant capacity of D. melanogaster. The antioxidant activity was evaluated using the activity of SOD and CAT and the content of MDA as indicators, and the survival time under acute oxidative damage caused by paraquat and hydrogen peroxide was measured. The results showed that high dose of $V$. coloratum polysaccharide significantly improved the fertility and lifespans of the female and male in the $\mathrm{F}_{1}-\mathrm{F}_{4}$ generation, reduced MDA content and increased SOD and CAT activities, and prolonged the survival time under acute oxidative damage caused by hydrogen peroxide and paraquat. Therefore, $V$. coloratum polysaccharide could improve the antioxidant capacity and vitality of $D$. melanogaster and promote their reproductive capacity. In addition, the antioxidant effect on female was stronger than male. The male to female ratio in the $\mathrm{F}_{1}-\mathrm{F}_{4}$ generations did not change much, and was overall close to $1: 1$.

The aging of $D$. melanogaster is closely related to the levels of free radicals, antioxidant capacity and methylation in the body. Excessive free radicals react with unsaturated fatty acids in the cell membrane, which can destroy the structures of biological macromolecules, such as DNA and proteins, which in turn leads to the occurrence of diseases and even death ${ }^{[23]}$. Paraquat is a fast-acting contact-kill herbicide with strong toxicity. It can generate superoxide anion free radicals, which in turn caused oxidative damage to the body. Hydrogen peroxide is a kind of active oxygen that can generate hydroxyl radicals through chemical reactions. It can participate in the metabolic response of living cells to produce functional decline, which further causes oxidative damage to the body (Dinis-Oliveira et al., 2008).

V. coloratum polysaccharide is a macromolecular substance with a variety of biologically active functions. When the polysaccharide was fed to D. melanogaster, it significantly affected the SOD and CAT activities and MDA content in D. melanogaster, suggesting that $V$. coloratum polysaccharide has a specific relationship with fertility, lifespan and aging of $D$. melanogaster. MDA is the decomposition product of lipid peroxide produced from polyunsaturated fatty acids in the cell membrane under the action of free radicals when $D$. melanogaster is stimulated by external stress. It will cause body aging when polymerized with large molecules, such as proteins and peptides, thereby influencing the longevity of D. melanogaster.

Both SOD and CAT have certain antioxidant activities, which can remove free radicals in the body and delay aging by reducing lipid peroxidation. With high concentration of $V$. coloratum polysaccharide, the MDA content was decreased sharply, and the antioxidant activities of SOD and CAT were significantly increased. This may be due to the participation of V. coloratum polysaccharide in complex biochemical reactions. It can stimulate $D$. melanogaster to increase the activities of SOD and CAT, and eliminate the MDA content produced in the body, so as to enhance the resistance and indirectly promote fertility and longevity of $D$. melanogaster.

\section{Conclusion}

In conclusion, our results showed that $V$. coloratum polysaccharide improved the antioxidant capacity and vitality of D. melanogaster and promoted their reproductive capacity in a dose-dependent manner. This study will provide a theoretical basis for the development of $V$. coloratum polysaccharide into health functional foods and medicines that delay aging, prolong life and improve fertility.

\section{Acknowledgement}

This work was supported by Science and Technology Guidance project of Longnan City (No. 201609) and Gansu Province University Innovation Capacity Enhancement Program (No. 2019A-190).

\section{References}

Brandt, A., \& Vilcinskas, A. (2013). The fruit fly Drosophila melanogaster as a model for aging research. Advances in Biochemical Engineering/ Biotechnology, 135(19), 63-77.http://dx.doi.org/10.1007/10_2013_193. PMid:23604209.

Chen, Y. Y., Hou, J. P., Huang, L., Khan, A., Xing, F. F., Zhang, X. H., Han, D. F., Yan, S. L., Cao, G. D., Jiao, Q. Y., Liu, D., Zhu, X., Hu, Q., \& Lou, H. X. (2020). Chemical constituents of Viscum coloratum (Kom.) Nakai and their cytotoxic activities. Natural Product Research, 27, 1-7. http://dx.doi.org/10.1080/14786419.20 20.1837816. PMid:33107346. 
Dinis-Oliveira, R. J., Duarte, J. A., Sánchez-Navarro, A., Remião, F., Bastos, M. L., \& Carvalho, F. (2008). Paraquat poisonings: mechanisms of lung toxicity, clinical features, and treatment. Critical Reviews in Toxicology, 38(1), 13-71. http://dx.doi.org/10.1080/10408440701669959. PMid:18161502.

Harman, D. (1981). The aging proces. Proceedings of the National Academy of Sciences of the United States of America, 78(11), 71247128. http://dx.doi.org/10.1073/pnas.78.11.7124. PMid:6947277.

Huang, H., \& Huang, G. (2020). Extraction, separation, modification, structural characterization, and antioxidant activity of plant polysaccharides. Chemical Biology \& Drug Design, 96(5), 1209-1222. http://dx.doi.org/10.1111/cbdd.13794. PMid:32959524.

Jana, S. C., Bettencourt-Dias, M., Durand, B., \& Megraw, T. L. (2016). Drosophila melanogaster as a model for basal body research. Cilia, 5(1), 22. http://dx.doi.org/10.1186/s13630-016-0041-5. PMid:27382461.

Jun, W., \& Yi, F. Z. (2007). Extraction and content determination of polysaccharides in Viscum coloratum. Academy of Traditional Chinese Medicine, 32(22), 2387-2390. PMID: 18257266.

Lamas, G. A., Navas-Acien, A., Mark, D. B., \& Lee, K. L. (2016). Heavy metals, cardiovascular disease, and the unexpected benefits of chelation therapy. Journal of the American College of Cardiology, 67(20), 2411-2418. http://dx.doi.org/10.1016/j.jacc.2016.02.066. PMid:27199065.

Li, Y. M., Chan, H. Y. E., Huang, Y., \& Chen, Z. Y. (2007). Green tea catechins upregulate superoxide dismutase and catalase in fruit flies.
Molecular Nutrition \& Food Research, 51(5), 546-554. http://dx.doi. org/10.1002/mnfr.200600238. PMid:17440995.

Rubin, R. M. (1988). Drosophila melanogaster as an experimental organism. Science, 240(4858), 1453-1459. http://dx.doi.org/10.1126/ science. 3131880

Stein, G. M., Edlund, U., Pfüller, U., Büssing, A., \& Schietzel, M. (1999). Influence of polysaccharides from Viscum album L.on human lymphocytes, monocytes and granulocytes in vitro [J]. Anticancer Research, 19(5B), 3907-3914. PMid:10628330.

Szurpnicka, A., Zjawiony, J. K., \& Szterk, A. (2019). Therapeutic potential of mistletoe in CNS-related neurological disorders and the chemical composition of Viscum species. Journal of ethnopharmacology, 231, 241-252. http://dx.doi.org/10.1016/j.jep.2018.11.025. PMid:30458281.

Peng, C., Chan, H. Y., Li, Y. M., Huang, Y., \& Chen, Z. Y. (2009). Black tea the aflavins extend the lifespan of fruits flies. Experimental Gerontology, 44(12), 773-783. http://dx.doi.org/10.1016/j.exger.2009.09.004. PMid:19770032.

Ye, W. B., Fan, L., \& Wang, Y. (2017). The protection mechanism of Viscum coloratum (Kom.) Nakai f.lutescens kitag fruit polysaccharides on acute alcoholic liver injury in mice. Anhui Nongye Daxue Xuebao, $44(2), 218-223$.

Yoo, J.-M., Park, K. I., \& Ma, J. Y. (2019). Anticolitic Effect of Viscum coloratum through Suppression of Mast Cell Activation. The American Journal of Chinese Medicine, 47(1), 203-221. http://dx.doi. org/10.1142/S0192415X19500101. PMid:30612453. 